\title{
Determinación del contenido de compuestos de cromo en el cemento portland por espectrofotometría de absorción atómica
}

Primera parte

DEMETRIO GASPAR-TEBAR, FERNANDO TRIVIÑO-VAZQUEZ

VICENTE ALVAREZ PUENTE

\section{R E S U M E N}

En el presente trabajo se da cuenta de un procedimiento para determinar el contenido de compuestos de cromo en el cemento portland por absorçión atómica, bięn por el método directo o por el de las adiciones patrones. La fusión se realiza con $\mathrm{LiBO}_{2}$ y la eliminación de interferencias por adición de La (III) (1\%).

Para el desarrollo del trabajo ha sido necesario averiguar dicho contenido por espectrofotocolorimetría del complejo formado por reacción del cromo presente en los cémentos, una vez pasado a $\mathrm{Cr}$ (VI), con la s-difenilcarbacida. Por otra parte se ha estudiado una variación del método propuesto sustituyendo la fusión alcalina con $\mathrm{Na}_{2} \mathrm{CO}_{3}$, o con la mezcla $\mathrm{Na}_{2} \mathrm{CO}_{3}-\mathrm{K}_{2} \mathrm{CO}_{3}$, por la fusión con $\mathrm{LiBO}_{2}$ que proporciona resultados menos dispersos y una desviación estándar estimada, una precisión y un coeficiente de variación menores.

Si bien la sensibilidad del método colorimétrico es mayor que la del método propuesto de absorción atómica, éste último es más rápido, con una precisión del mismo orden y, además, permite analizar cuantitativamente los compuestos de cromo siguiendo la marcha que normalmente se utiliza para los demás constituyentes del cemento por absorción atómica. Se hace un estudio estadístico, calculando la desviación estándar estimada, la precisión y el coeficiente de variación de los resultados obtenidos en diversos cementos y clínkeres de cemento portland españoles.

\section{INTRODUCCION}

Es sabido que la presencia de constituyentes minoritarios (elementos traza) en cualquier substancia o producto, tanto natural como artificial, juega un papel importante en cuanto a sus propiedades; de aquí, la necesidad de detectar la naturaleza y concentración de estos elementos y por consiguiente, poner a punto, caso de que no existan, técnicas de trabajo apropiadas para su determinación. 
Entre los constituyentes minoritarios del cemento portland merece especial atención el cromo por actuar tanto sobre las propiedades del cemento (variación de las resistencias mecánicas a corto y largo plazo, cambios en la coloración de los cementos blancos, control de la corrosión de las armaduras del hormigón, etc.) como por sus consecuencias tóxico-contaminantes.

La presencia del cromo en los cementos (20-100 ppm) (1) (2) es debida, fundamentalmente, a las materias primas utilizadas en su fabricación, en donde se encuentra como impureza. Valle (1) considera que también se produce un aporte secundario como consecuencia del desgaste de los elementos moledores y de los materiales refractarios, aporte que según Keil (2) no ha podido ser comprobado.

En el presente trabajo se expone un método espectrofotocolorimétrico para llevar a cabo dicha determinación y en una futura segunda parte se describirá otro método, estudiado por nosotros, que utiliza la espectrofotometría de absorción atómica.

\section{BREVES CONSIDERACIONES SOBRE EL CROMO (3) a (y)}

Es sabido que el cromo, después del cobalto y del níquel, es el elemento que presenta más tendencia a formar compuestos complejos, pese a lo cual es frecuente encontrar cromo (con números de valencia $\mathrm{O}$ a VI) en compuestos simples (3).

De estos estados, la especie que existe normalmente como catión muy estable es el cromo (III), que permanece en disolución incluso en medios oxidantes y ácidos. El cromo (II) es un reductor muy enérgico que tiene gran capacidad para formar complejos coloreados con índice de coordinación 4 ó 6 ; esta propiedad se aprovecha para su determinación cuantitativa por colorimetría.

Los estados de valencia (IV) y (V) sólo se detectan en los pasos intermedios de los procesos redox, son metaestables, y se transforman en cromo (III) o cromo (VI) según las características del medio.

En el estado de cromo (VI) hay tres especies principales, $\mathrm{CrO}_{3}, \mathrm{CrO}_{4}{ }^{2-}$ y $\mathrm{Cr}_{2} \mathrm{O}_{7}{ }^{2-}$; también se conoce la existencia de peróxidos, perácidos y percromatos estables. La existencia de $\mathrm{CrO}_{4}{ }^{2-}$ ó $\mathrm{Cr}_{2} \mathrm{O}_{7}{ }^{2-}$ depende de la acidez del medio de reacción; así, en medio básico el anión estable es el cromato que comunica a la disolución una coloración amarilla, la cual se aprovecha para su determinación cuantitativa por colorimetría; por el contrario, en medio ácido el anión estable es el dicromato, y la determinación cuantitativa normal de este anión se basa en sus propiedades oxidantes.

\section{ESTUDIO BIBLIOGRAFICO}

El cromo presente en los silicatos naturales o artificiales se puede determinar cuantitativamente tanto por técnicas químicas (gravimétricas y volumétricas) como por técnicas instrumentales.

Voinnovitch y colaboradores (8), así como Bennet y Hawley (9) proponen un método volumétrico en el análisis de silicatos (cuando la concentración de cromo es superior al $1 \%$ ) basado en la transformación de los compuestos de cromo en $\mathrm{Cr}_{2} \mathrm{O}_{7}{ }^{2-}$, que se deter- 
mina por una yodimetría (10) o por reducción con un exceso de una disolución tipo de $\mathrm{Fe}$ (II), valorando el exceso de $\mathrm{Fe}$ (II) no oxidado por el $\mathrm{Cr}$ (VI) por medio de una permanganimetría (11) y otros.

Jakob (12) describe un método gravimétrico para el análisis de rocas y suelos, útil para concentraciones de cromo superiores al $0,05 \%$; el cromo (III) se precipita al estado de $\mathrm{Cr}(\mathrm{OH})_{3}$, el cual se seca, calcina y pesa.

Burriel (13) para concentraciones superiores a $10 \mathrm{ppm}$ describe un método espectrofotométrico de emisión (se utiliza la línea $425,4 \mathrm{~nm}$ ).

La espectrografía de arco ha sido utilizada por Valle (1) y por Pinta (14), quien también emplea la polarografía consiguiendo una sensibilidad de $1 \mathrm{ppm}$.

Los métodos más utilizados en la práctica para determinar concentraciones pequeñas de cromo son los espectrofotocolorimétricos; así Jakob (12), Bennet y. Hawley (9) y Pinta (14) proponen la determinación del ion $\mathrm{CrO}_{4}{ }^{2-}$ (coloración amarilla) a $\mathrm{pH}$ mayor de $9 \mathrm{y}$ Fischer-Peters (11) del ion $\mathrm{Cr}_{2} \mathrm{O}_{7}{ }^{2-}$.

Duche (15) utiliza un método basado en la medida de la coloración roja del complejo formado por reacción entre el cromo (VI) y la 2-metil-8-quinoleína, a una $\lambda$ de $580 \mathrm{~nm}$.

En el método descrito por Bennet y Hawley (9) se determina la intensidad del color rojo anaranjado formado entre el cromo (III) y la complexona (II).

La determinación de la intensidad del color rojo-violeta del complejo formado por reacción del cromo (VI) con la s-difenilcarbacida, en medio $\mathrm{H}_{2} \mathrm{SO}_{4} \cdot 0,2 \mathrm{~N}$, a una longitud de onda de $540 \mathrm{~nm}$, ha sido utilizada por Charlot (16), Stand. Meth. of Chem. Anal. (17), Hartford (7) y otros en diversos casos; por Valle (1), Flatt y Cusani (18) para el análisis de cementos y por Voinovitch y colaboradores (8), Bennet y Hawley (9) para el análisis de silicatos. El mecanismo de esta reacción ha sido estudiado por Bose (19), Pflaum y. Howick (20), Linchestein y Allen (21), Balt y Van Dalen (22).

El complejo formado, con un coeficiente de extinción molar de 31.400 para una solución molar de $\mathrm{K}_{2} \mathrm{Cr}_{2} \mathrm{O}_{7}$ (7), es estable en medio $\mathrm{H}_{2} \mathrm{SO}_{4} .0,2 \mathrm{~N}$, (1); la presencia de oxidantes y reductores enérgicos descomponen a dicho complejo.

Marczenko (23) recoge diversos métodos espectrofotométricos para determinar el cromo - de un modo especial el de la s-difenilcarbacida, del cromato y del EDTA- estudiando las técnicas de separación por extracción y precipitación. El cromo ha sido determinado por el método de la s-difenilcarbacida en hierro y acero, aleaciones, en diversos metales, cemento, vidrio, minerales, etc.

La absorción molar del complejo coloreado del $\mathrm{Cr}$ (VI) con la s-difenilcarbacida es $4,17 \times$ $\times 10^{4}$ a $\lambda$ máxima $=546 \mathrm{~nm}$; la intensidad del color depende de la calidad del reactivo s-difenilcarbacida utilizado.

Kolthoff y colaboradores (24) describen un método espectrofotométrico para determinar mezclas de permanganato y dicromato, midiendo la absorbancia a $\lambda$ de $440 \mathrm{~nm}$ y $545 \mathrm{~nm}$; el método descrito se aplica para determinar cromo y manganeso en hierro y acero.

Charlot (16) recopila una serie de posibilidades de extracción del complejo con diversos disolventes.

Katz, Mc Nabb y Hazel (25) describen un método para extraer el cromo de disoluciones sulfúricas con metil-isobutilcetona (MIBK); método que ha sido aplicado por Valle (1) al caso de cementos, llegando a la conclusión de que la extracción en este caso no es cuantitativa. 
Degré (26) aplica el autoanalizador Technicon para automatizar el análisis elemental cuantitativo por espectrometría de absorción atómica de diferentes materias primas y productos de la industria del cemento. Señala, entre otros, el contenido de cromo en un clínker, una escoria y una bauxita; la llama utilizada es aire-acetileno.

Feldman y Purly (27) estudian la posibilidad de aplicar la espectrofotometría de absorción atómica para determinar la concentración de cromo en muestras metálicas, seleccionando entre las posibles líneas analíticas $(3.578,7 \AA, 3.593,5 \AA, 3.605,3 \AA, 4.254,4 \AA, 4.274,8$ $\AA, 4.289,7 \AA$ ) la línea $3.578,7 \AA$ por ser la que proporciona mayor sensibilidad relativa cuando se utiliza una llama de tipo aire-acetileno.

Con la llama óxido nitroso-acetileno se puede utilizar la línea 4.353,4 $\AA$, aun cuando, en general, dada la naturaleza de metal pesado del cromo, se obtienen mejores resultados trabajando con llamas de tipo aire-acetileno.

Tenoutasse (28) recopila la longitud de onda, la resolución, el límite de detección y la sensibilidad de diferentes elementos que presentan interés para sus trabajos relacionados con el análisis de cementos por espectrofotometría de absorción atómica; para el caso del cromo son $357,9 \mathrm{~nm} ; 0,005 \mu \mathrm{g} / \mathrm{ml} ; 0,15 \mu \mathrm{g} / \mathrm{ml} 1 \%$ y 2 , respectivamente.

Fifield y Kealy (29) mencionan que para determinar el cromo por espectrofotometría de absorción atómica trabajando con llama aire-acetileno, se debe utilizar la línea espectral de $359 \mathrm{~nm}$; el límite de detección es 0,01 ppm.

Skoog y West (30) enumeran el límite de detección y la longitud de onda de los picos de absorción más sensibles de algunos de los elementos más comunes; así para el cromo son $0,05 \mu \mathrm{g} / \mathrm{ml}$, concentración que absorbe $1 \%$ de $P_{0}$ que corresponde a una transmitancia de $99 \%$ o a una absorbancia de 0,004 y $357,9 \mathrm{~nm}$.

\section{PARTE EXPERIMENTAL}

\subsection{Determinación del contenido de compuestos de cromo en el cemento portland por espectrofotocolorimetría}

Con el fin de poder determinar el contenido de compuestos de cromo en el cemento portland por espectrofotometría de absorción atómica, objeto del presente trabajo, se ha efectuado en una primera parte la determinación de dicho contenido en diversas muestras de cementos industriales por espectrofotocolorimetría, apoyándonos, para ello, en los trabajos efectuados hasta el momento. De este modo se pretende realizar, a la vez, un estudio comparativo y disponer de muestras con contenidos conocidos de cromo.

\subsection{Equipo y condiciones de trabajo}

Se ha usado un espectrofotómetro Beckman, modelo D.U., equipado con lámpara de wolframio; las células empleadas han sido de cuarzo y la longitud de onda $540 \mathrm{~nm}$. 


\subsection{Disoluciones y reactivos empleados}

a) Disolución reactivo de s-difenilcarbacida (1,5 difenilcarbacida).

Se disuelven 0,25 g del reactivo (1) (23) (14) (17) s-difenilcarbacida (Merck, p.a.), en $50 \mathrm{ml}$ de acetona y a continuación se llevan por dilución, con agua destilada o de pureza similar, a $100 \mathrm{ml}$.

La concentración de reactivo es por consiguiente $0,5 \%$ en un medio acetona-agua $(1: 1)$ (1) (17) (31).

La disolución se prepara en el momento de utilizarla.

b) Disolución patrón de cromo (VI), con una concentración de 0,1 g de cromo por litro de disolución.

La disolución patrón de cromo (VI), con una concentración de $0,1 \mathrm{~g}$ de cromo/litro, se ha preparado disolviendo en agua desionizada, exenta de reductores, 0,282 g g de $\mathrm{K}_{2} \mathrm{Cr}_{2} \mathrm{O}_{7}$ Merck, p.a., previamente secado a $140^{\circ}-150^{\circ} \mathrm{C}$, que se llevan a $1.000 \mathrm{ml}$.

c) Metaborato de litio.

Se ha utilizado $\mathrm{LiBO}_{2}$, Phaxe, de calidad Atomic Absorption.

d) Carbonato sódico potásico anhidro, Probus reactivo, exento de cromo.

\subsection{Muestras de cemento estudiadas}

Se han estudiado 10 muestras de cemento portland, fabricado por la industria española, que responden a la composición química que figura en la tabla 1.

TABLA 1

Composición química de las muestras utilizadas

\begin{tabular}{|c|c|c|c|c|c|c|c|c|c|c|c|}
\hline \multirow{2}{*}{ Determinación } & & \multicolumn{10}{|c|}{ Muestra $n .^{\circ}$} \\
\hline & & 1 & 2 & 3 & 4 & 5 & 6 & 7 & 8 & 9 & 10 \\
\hline Pérdida por calcinación ... & P.F. & 1,0 & 2,2 & 3,2 & 2,1 & 1,9 & 2.6 & 1,7 & 2,8 & 3,4 & 2,9 \\
\hline Residuo insoluble ... ... .. & R.I. & 2,7 & 2,6 & 1,6 & 0,9 & 0,8 & 1,7 & $2, \overline{2}$ & 2,4 & 2,1 & 8,3 \\
\hline Dióxido de silicio ... ... .. & $\mathrm{SIO}_{2}$ & 22,2 & 20,2 & 19,2 & 18,7 & 19,8 & 17,8 & 18,3 & 17,6 & 17,4 & 21,0 \\
\hline Oxido alumínico $\ldots \ldots \ldots$ & $\mathrm{A}_{2} \mathrm{O}_{3}$ & 4,7 & 5,4 & 6,0 & 5,4 & 6,6 & 7,7 & 8,1 & 5,6 & 5,2 & 6,0 \\
\hline Oxido férrico $\ldots \begin{array}{llll} & \ldots & \ldots & \ldots\end{array}$ & $\mathrm{Fe}_{2} \mathrm{O}_{3}$ & 3,7 & 2,6 & 2,2 & 1,9 & 1,8 & 2,1 & 2,2 & 4,7 & 4,8 & 3,0 \\
\hline Oxido cálcico $\ldots \begin{array}{llll} & \ldots & \ldots & \ldots\end{array}$ & $\mathrm{CaO}$ & 62,4 & 62,6 & 63,4 & 63,5 & 61,6 & 61,6 & 61,3 & 60,4 & 60,8 & 54,2 \\
\hline Oxido magnésico $\ldots . . . .$. & $\mathrm{MgO}$ & 1,6 & 1,7 & 1,3 & 1,7 & 1,8 & 1,4 & 1,1 & 1,7 & 1,8 & 1,2 \\
\hline Trióxido de azufre $\ldots \ldots$ & $\mathrm{SO}_{3}$ & 1,7 & 1,6 & 2,6 & 3,9 & 3,9 & 4,1 & 3,2 & 3,2 & 3,9 & 1,7 \\
\hline Oxido sódico $\ldots \ldots \ldots \ldots$ & $\mathrm{Na}: \mathrm{O}$ & 0,2 & 0,1 & 0,4 & s.d. & s.d. & s.d. & s.d. & s.d. & s.d. & s.d. \\
\hline \multirow[t]{2}{*}{ 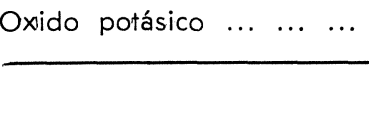 } & $\mathrm{K}_{2} \mathrm{O}$ & 0,3 & 0,8 & 0,9 & s.d. & s.d. & s.d. & s.d. & s.d. & s.d. & s.d. \\
\hline & Suma & 100,5 & 99,8 & 100,8 & 98,1 & 98,2 & 99,0 & 98,1 & 98,4 & 99,4 & 98,3 \\
\hline
\end{tabular}


La composición química se ha determinado de acuerdo con la técnica de trabajo descrita en el apartado 2.7. del PCCH-64 (32) y el contenido de elementos alcalinos, $\mathrm{Na}$ (I) y $\mathrm{K}$ (I), por espectrofotometría de llama. Los resultados, referidos a la muestra seca a $105^{\circ}-110^{\circ} \mathrm{C}$, se han expresado, en $\%$ en peso, como óxidos excepto la pérdida por calcinación y el residuo insoluble.

\subsection{Técnica de trabajo}

Las operaciones fundamentales para determinar el contenido de compuestos de cromo en el cemento portland las hemos reunido en los siguientes grupos:

a) Preparación de la muestra que comprende disolución de la misma, oxidación de los compuestos de $\mathrm{Cr}$ (III) a $\mathrm{Cr}$ (VI) y eliminación de interferencias.

\section{Disolución}

La disgregación de las muestras de cemento portland estudiadas se ha hecho, por una parte, con la mezcla $\mathrm{Na}_{2} \mathrm{CO}_{3}-\mathrm{K}_{2} \mathrm{CO}_{3} \mathrm{y}$, por otra, con $\mathrm{LiBO}_{2}$, ya que éste se utiliza en la fusión del cemento, crudos y materias primas de la industria del cemento para determinar su composición química por espectrofotometría de absorción atómica.

El empleo del $\mathrm{LiBO}_{2}$ para la disgregación del cemento portland, crudos y materias primas, con el fin de analizarlos por espectrofotometría de absorción atómica (33) (34) (35), presenta diversas ventajas como son: utilizar una pequeña cantidad de fundente, poco tiempo y baja temperatura para realizar la fusión, uso de crisoles de grafito que se pueden utilizar para hacer de 60 a 100 fusiones; el producto fundido es soluble en ácidos minerales proporcionando disoluciones estables y transparentes, no introduce cationes comunes como son $\mathrm{K}$ (I) y $\mathrm{Na}$ (I), etc.

Valle (1) ha utilizado como fundente del cemento $\mathrm{Na}_{2} \mathrm{~B}_{4} \mathrm{O}_{7}$ y $\mathrm{Na}_{2} \mathrm{CO}_{3}$ para los crudos y arcillas, fundente recomendado por Jakob para el análisis de silicatos (12), el cual ha probado que ambos fundentes no producen interferencias en la determinación espectrofotocolorimétrica del cromo en cementos, teniendo en cuenta las cantidades y condiciones de empleo.

\section{Oxidación}

Como agente oxidante del $\mathrm{Cr}$ (III) a $\mathrm{Cr}$ (VI) se ha utilizado $\mathrm{K}_{2} \mathrm{~S}_{2} \mathrm{O}_{8}$ en presencia de una pequeña cantidad de $\mathrm{Ag}$ (I), procedente de una disolución acuosa de $\mathrm{AgNO}_{3}$. Por medio de esta técnica se consigue la oxidación cuantitativa del $\mathrm{Cr}$ (III) a $\mathrm{Cr}$ (VI) y del posible $\mathrm{Fe}$ (II) a $\mathrm{Fe}$ (III). La eliminación del exceso de oxidante es fácil, ya que basta para ello hervir la disolución; se produce la transformación del $\mathrm{S}_{2} \mathrm{O}_{8}{ }^{2-}$ en $\mathrm{SO}_{4}{ }^{2-}$ y se elimina $\mathrm{O}_{2}$.

\section{Eliminación de interferencias}

En la determinación del cromo (VI) en los cementos portland, por espectrofotocolorimetría del complejo formado con la s-difenilcarbacida, interfiere el catión $\mathrm{Fe}$ (III) que se elimina del medio por precipitación al estado de $\mathrm{Fe}(\mathrm{OH})_{3}$ y filtrado posterior.

Los iones $\mathrm{Mg}$ (II), $\mathrm{Ag}$ (I), $\mathrm{Cu}$ (II), $\mathrm{Pb}$ (II), Co (II) y $\mathrm{Ni}$ (II) interfieren en grado pequeño; sin embargo, el Mo (VI) y el V (V) interfieren cuando se encuentran en grandes cantidades. 
Conjuntamente con el $\mathrm{Fe}$ (III) se eliminan en el cemento, también al estado de hidróxidos, los cationes Al (III), Ti (IV) y Sr (II).

b) Formación del complejo coloreado

La formación del complejo coloreado tiene lugar por reacción del $\mathrm{Cr}$ (VI) con la disolución reactivo de la s-difenilcarbacida en medio $\mathrm{H}_{2} \mathrm{SO}_{4} \cdot 0,2 \mathrm{~N}$.

c) Medida de la transmitancia o absorción de la disolución que contiene el complejo y de otra disolución $€ n$ blanco, que se utiliza como referencia, por medio de un espectrofotocolorímetro

d) Tratamiento y expresión de los resultados obtenidos

€) Técnica operatoria

La fusión con la mezcla $\mathrm{Na}_{2} \mathrm{CO}_{3}-\mathrm{K}_{2} \mathrm{CO}_{3}$ se ha realizado de acuerdo con la técnica descrita en (1) (36) (37) (38) (33) y otros. Se pesa aproximadamente $1 \mathrm{~g}$ de cemento, con una precisión de $\pm 0,0001 \mathrm{~g}$, y se mezcla íntimamente con $3,5 \mathrm{~g}$ de fundente. La mezcla se coloca en un crisol de plaíino y se somete a la acción del calor hasta fusión completa. El producto fundido se pasa a una cápsula de platino que tiene $\mathrm{H}_{2} \mathrm{SO}_{4}$ conc. caliente, el cual disuelve dicho produc:o, dejando un precipitado de ácido silícico que se separa por filtración y se purifica por lavado.

La fusión con $\mathrm{LiBO}_{2}$ se ha realizado en crisol de grafito, utilizando aproximadamente $0,3 \mathrm{~g}$ de cemento, pesados con una precisión de $\pm 0,0001 \mathrm{~g}$, que se mezclan íntimamente en un mortero de ágata con $0,3 \mathrm{~g}$ de $\mathrm{LiBO}_{2}$. $\mathrm{La}$ mezcla se introduce en un crisol de grafito de fondo plano, se cubre con $0,15 \mathrm{~g}$ de fundente y se tapa con una rodaja de grafito; a continuación se somete el conjunto a la acción del calor, en corriente de nitrógeno, en un horno a $1.000 \mathrm{C}$ durante 4-5 minutos; transcurrido este tiempo, se destapa el crisol, se le imprime un movimiento de rotación hasta formar una perla y se prosigue la calefacción durante o`ros 4-5 minutos. Por último se vacía el contenido del crisol sobre $25 \mathrm{ml}$ de $\mathrm{H}_{2} \mathrm{SO}_{4} .4 \mathrm{~N}$ contenido en un vaso de precipitados de $250 \mathrm{ml}$ de forma alta y se agita, con un agitador magnético, hasta conseguir la disolución de la perla; normalmente se forma un precipitado blanco de sulfato cálcico que, junto a las partículas de grafito, se separa por filtración en frío.

Una vez llevada a disolución la muestra de cemento por disgregación con $\mathrm{Na}_{2} \mathrm{CO}_{3}-\mathrm{K}_{2} \mathrm{CO}_{3}$ o con $\mathrm{LiBO}_{2}$ y separada por filtración la fase sólida, se le añaden al filtrado $0,3 \mathrm{~g}$ de $\mathrm{K}_{2} \mathrm{~S}_{2} \mathrm{O}_{8}$ y $0,3 \mathrm{ml}$ de una disolución acuosa $0,1 \mathrm{~N}$ de $\mathrm{AgNO}_{3}$; a continuación se calienta suavemente la disolución sin que hierva, a la vez que se agita, en baño maría durante 2030 minutos y se añade, en frío, una disolución de $\mathrm{NaOH}$ hasta conseguir un $\mathrm{pH}$ comprendido entre 7 y 9 ; se calienta en baño de vapor y se separa el precipitado formado de hidróxidos, en cali:nte, por filtración y, finalmente, se purifica por lavado.

El filtrado, junto con las aguas de lavado, se neutraliza con $\mathrm{H}_{2} \mathrm{SO}_{4}$ dil. hasta $\mathrm{pH}=7$, se concentra y se pasa a un matraz aforado de $125 \mathrm{ml}$ al que se añaden $12,5 \mathrm{ml}$ de $\mathrm{H}_{2} \mathrm{SO}_{4}$. $2 \mathrm{~N}$ y $7 \mathrm{ml}$ de la disolución reactivo de s-dif enilcarbacida; a continuación se enrasa el matraz con agua desionizada, se espera de 20 a 30 minutos y se procede a la lectura espectrofotocolorimétrica.

A partir de la lecıura espectrofotocolorimétrica y haciendo uso de la gráfica de calibrado se determina el contenido de cromo que se expresa en ppm. 


\section{RESULTADOS}

\subsection{Estudio del complejo Cr (VI)-s-difenilcarbacida}

a) Estabilidad

Para determinar la estabilidad del complejo cromo (VI)-s-difenilcarbacida a lo largo del tiempo (325 minutos) se ha realizado la medida del \% de transmitancia en 7 disoluciones que tienen la concentración de cromo (VI) que se señala en la figura 1, comprendida entre $0,02 \mathrm{mg} / l$ y $0,40 \mathrm{mg} / l$. Los resultados obtenidos han puesto de manifiesto:

- que el complejo es estable en el período de tiempo estudiado;

- que las lecturas se deben hacer transcurridos 20/30 minutos.

Las lecturas se han hecho en las 7 disoluciones mencionadas de 10 en 10 minutos, hasta 50 minutos, y a 110,290 y 325 minutos.

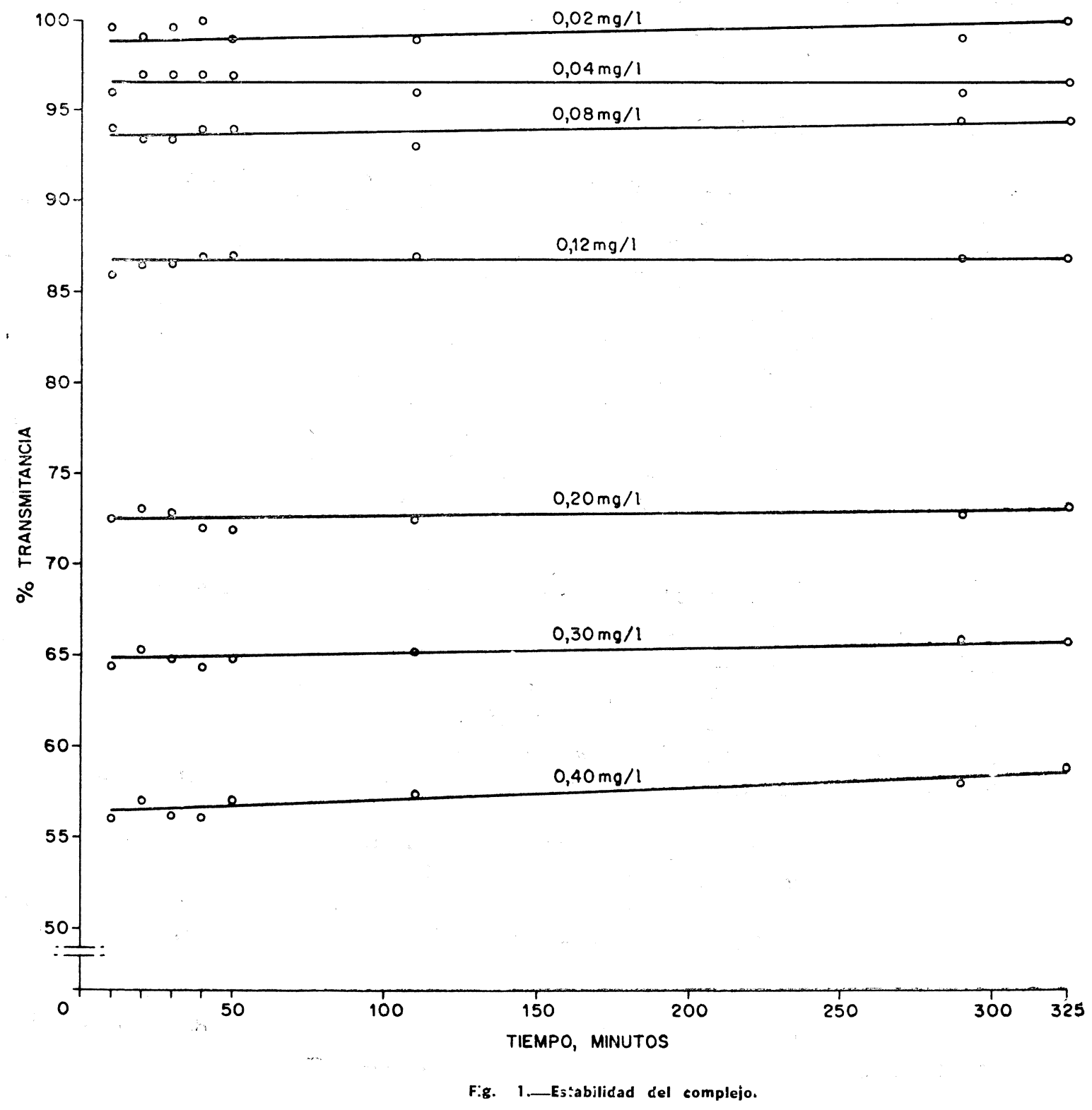


Por otra parte, en tres disoluciones con una concentración de $0,02 \mathrm{mg} / \mathrm{l}, 0,20 \mathrm{mg} / \mathrm{l}$ y 0,40 $\mathrm{mg} / l$ de cromo se ha determinado el $\%$ de transmitancia, además, a 48 y 72 horas; los valores obtenidos se incluyen en la tabla 2 .

TA B L A 2

Estabilidad del complejo. Influencia del tiempo

\begin{tabular}{|c|c|c|c|}
\hline \multirow{2}{*}{ Disolución, $\mathbf{m g} / \boldsymbol{l}$ de cromo } & \multicolumn{3}{|c|}{ Transmitancia, \% } \\
\cline { 2 - 3 } & inicial & $\mathbf{2 4 \text { horas }}$ & $\mathbf{4 8 \text { horas }}$ \\
\hline 0,02 & 97,0 & 96,8 & 99,0 \\
0,20 & 73,0 & 73,5 \\
0,40 & 56,0 & 58,5 & 59,0 \\
\hline
\end{tabular}

El medio es $\mathrm{H}_{2} \mathrm{SO}_{4} \cdot 0,2 \mathrm{~N}$. La cantidad de disolución reactivo de s-difenilcarbacida adicionada ha sido $10 \mathrm{ml}$.

Todas las determinaciones se han hecho en la atmósfera del laboratorio; la adición de $0,250 \mathrm{~g}$ de $\mathrm{K}_{2} \mathrm{~S}_{2} \mathrm{O}_{8}$ y $0,3 \mathrm{ml}$ de una disolución acuosa $0,1 \mathrm{~N}$ de $\mathrm{AgNO}_{3}$, produce una rápida decoloración del complejo.

b) Sensibilidad de la reacción

El límite de detección del método espectrofotocolorimétrico, en las condiciones de trabajo presentes, es de $0,02 \mathrm{mg} / l$, ya que para esta concentración se alcanza prácticamente el valor $100 \%$ para la transmitancia.

En la gráfica de la figura 2 se puede apreciar que para una variación de $\pm 0,01 \mathrm{mg} / \mathrm{l}$ se obtiene otra de $\pm 0,5 \%$ de transmitancia; no cumpliéndose la ley de Bouguer-LambertBeer para concentraciones inferiores a $0,02 \mathrm{mg} / l$ de cromo y superiores a $0,40 \mathrm{mg} / \mathrm{l}$.

c) Influencia de la cantidad de la disolución reactivo de s-difenilcarbacida

La cantidad de disolución reactivo que se utiliza, normalmente, €s de $1 \mathrm{ml}$ para concentraciones de cromo variables, p.e., para 5 a $50 \mathrm{ppm}$ (17), 2 a $15 \mu \mathrm{g}(16), 0,2$ a $15 \mu \mathrm{g}$ (7), $40 \mu \mathrm{g}(23), 1$ a $500 \mu \mathrm{g}$ (14), 0,2 a $0,5 \mathrm{ppm}$ de cromato (31); de $10 \mathrm{ml}$ de dicho reactivo para 10 a $100 \mathrm{ppm}$ en el caso de cementos (1), silicatos (8) (9), aceros y hierro electrolítico (17).

La reacción de formación del complejo, según Bose, tiene lugar entre 2 iones-gramo de cromo y 3 moles de s-difenilcarbacida, por lo que para $3 \mathrm{mg}$ de cromo se necesitarán, teóricamente, $10 \mathrm{ml}$ de disolución reactivo.

De aquí que se hayan utilizado, en una primera etapa, $10 \mathrm{ml}$ de la disolución reactivo y realizado, posteriormente, un estudio comparativo empleando 5 y $10 \mathrm{ml}$ para las concentraciones de cromo que se recogen en la tabla 3 , habiendo obtenido valores medios de transmitancia (\%), correspondientes a 10 lecturas concordantes, precisos (tabla 3); la mayor dispersión se tiene para la disolución de $0,02 \mathrm{mg} / l$ de cromo, que se encuentra, como se mencionó en el apartado anterior, en el límite de detección. 
Tabla 3

Influencia de la cantidad de disolución reactivo

\begin{tabular}{|c|c|c|}
\hline \multirow{2}{*}{ Cromo, $\mathrm{mg} / \mathrm{l}$} & \multicolumn{2}{|c|}{ Transmitancia, $\%$} \\
\hline & $5 \mathrm{ml}$ & $10 \mathrm{ml}$ \\
\hline 0,02 & 99,5 & 97,0 \\
\hline 0,04 & 96,0 & 95,7 \\
\hline 0,08 & 94,0 & 94,5 \\
\hline 0,12 & 86,0 & 87,0 \\
\hline 0,20 & 73,0 & 73,5 \\
\hline 0,30 & 64,0 & 64,0 \\
\hline 0,40 & 56,0 & 56,0 \\
\hline
\end{tabular}

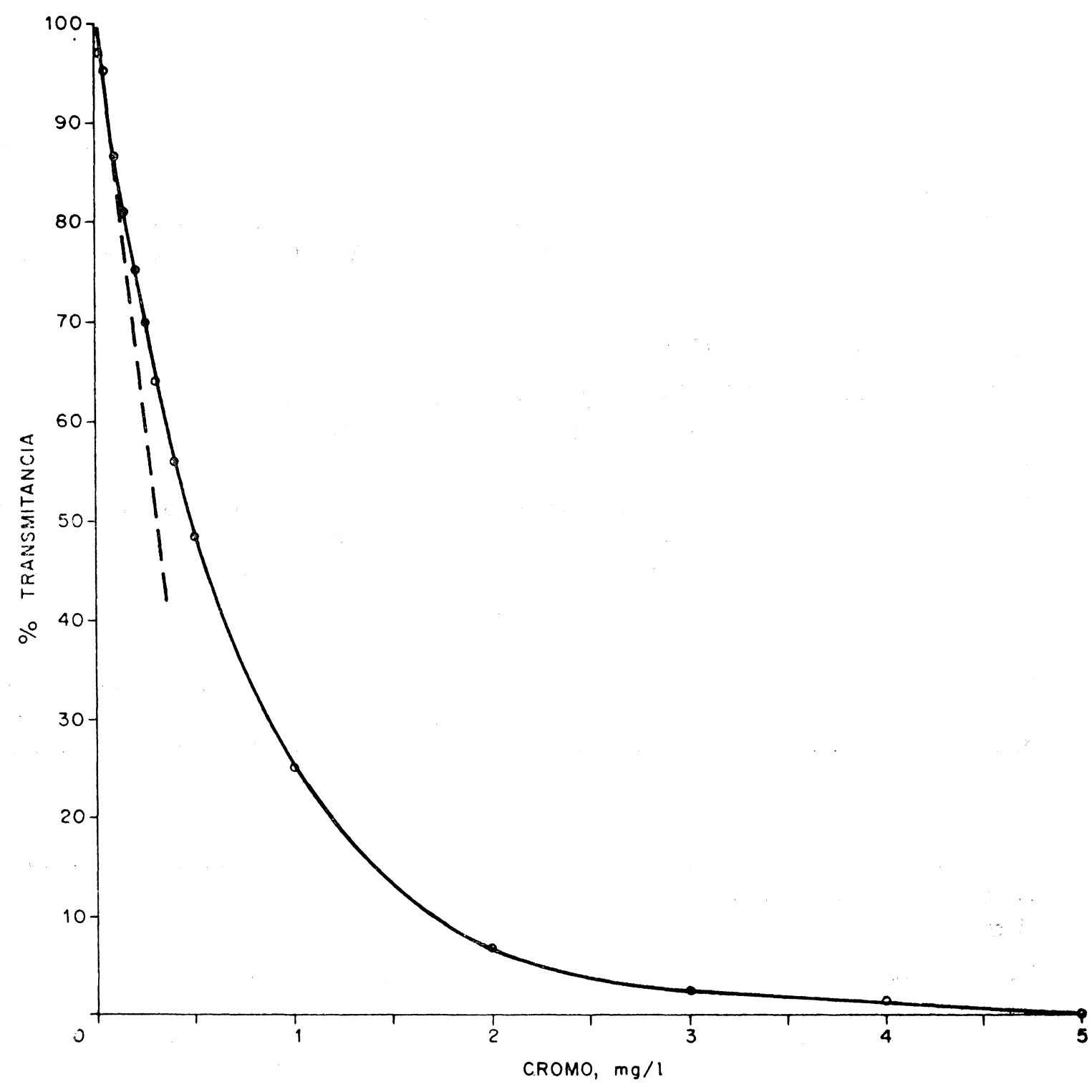

Fig. 2.-Gráfica de calibrado: $\%$ transmitancia-cromo, $\mathbf{m g} / l$. 


\subsection{Obtención de la gráfica de calibrado}

Para obtener la gráfica de calibrado se prepararon, a partir de la disolución patrón de cromo (VI), dos series de disoluciones acuosas con una concentración de cromo comprendida entre $0,50 \mathrm{mg} / l$ y $5,00 \mathrm{mg} / \mathrm{l}$ la primera, y $0,02 \mathrm{mg} / l$ y $0,40 \mathrm{mg} / l$ la segunda, a las que se ha adicionado $\mathrm{H}_{2} \mathrm{SO}_{4} .2 \mathrm{~N}$ hasta conseguir que el medio fuese $0,2 \mathrm{~N}$. A continuación se añade la disolución reactivo de s-difenilcarbacida y se lleva a $100 \mathrm{ml}$; al cabo de 20-30 minutos se determina la transmitancia de dicha disolución y de otra disolución en blanco que se utiliza como referencia.

Las cantidades adicionadas de la disolución reactivo de s-difenilcarbacida han sido 5 y 10 $\mathrm{ml}$ para la primera serie y $10 \mathrm{ml}$ para la segunda.

Los resultados obtenidos, valor medio de 10 lecturas distintas precisas (concordantes), representados en las figuras 2 y 3 , se incluyen en la tabla 4 .

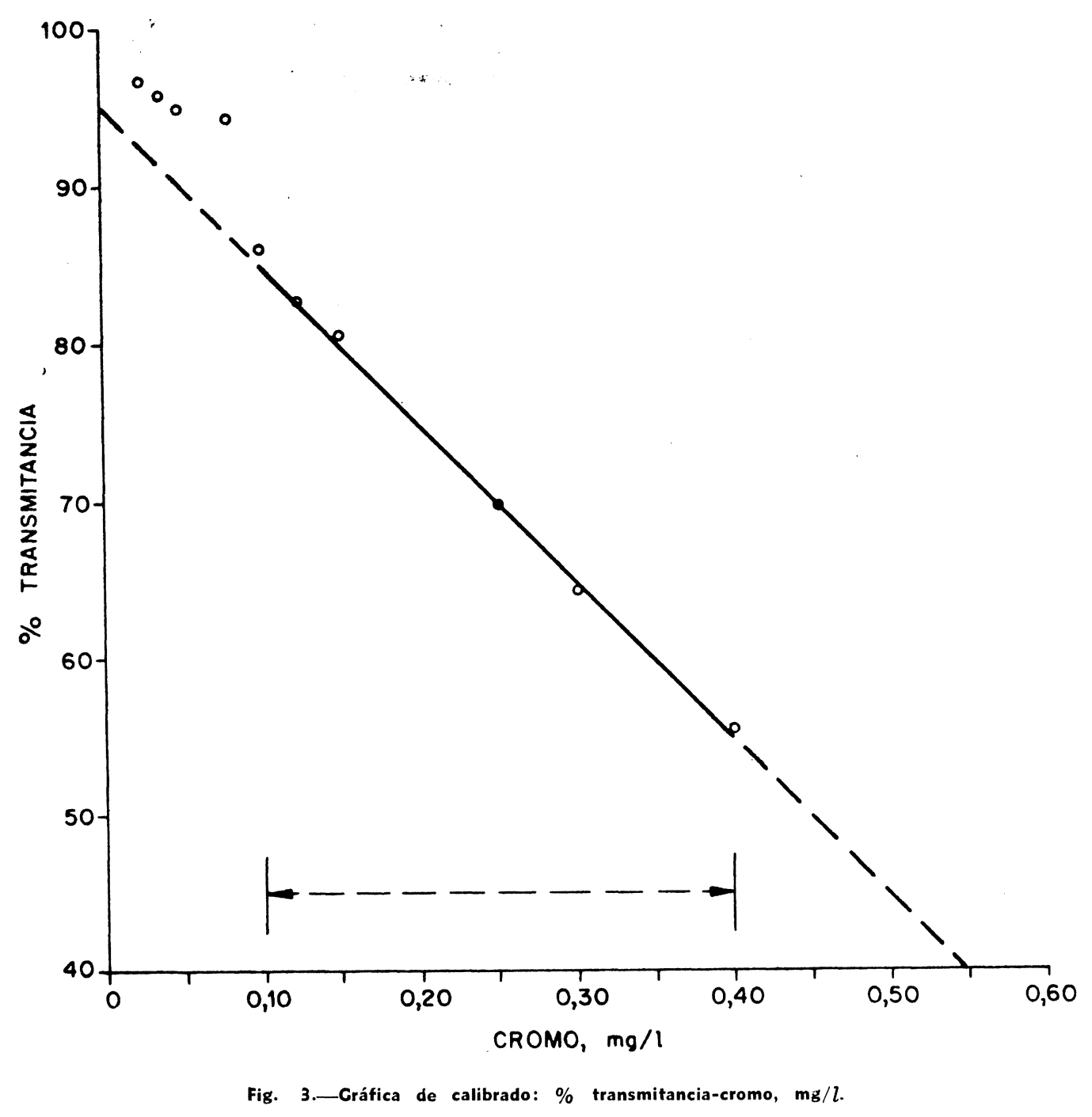

En el tramo recto de la figura 3, correspondiente al intervalo de concentraciones de cromo comprendido entre 0,10 y $0,40 \mathrm{mg} / l$, se cumple la ley de Bouguer-Lambert-Beer. De aquí que en la parte experimental se haya utilizado una cantidad de muestra que proporcione concentraciones comprendidas en dicho intervalo, bien directamente o por dilución. 
T A B L A 4

Transmitancia (\%) del complejo cromo-difenilcarbacida de disoluciones tipo de cromo (VI)

\begin{tabular}{|c|c|c|c|}
\hline \multicolumn{2}{|c|}{ Disolución } & \multirow{2}{*}{$\begin{array}{c}\text { Concentración de cromo } \\
\mathrm{mg} / \mathrm{l}\end{array}$} & \multirow{2}{*}{ Transmitancia, \% } \\
\hline serie & n.० & & \\
\hline \multirow{6}{*}{ I } & 1 & 5,00 & 0,0 \\
\hline & 2 & 4,00 & 1,0 \\
\hline & 3 & 3,00 & 2,0 \\
\hline & 4 & 2,00 & 7,0 \\
\hline & 5 & 1,00 & 26,0 \\
\hline & 6 & 0,50 & 49,0 \\
\hline \multirow{10}{*}{11} & 7 & 0,40 & \multirow{10}{*}{95,7} \\
\hline & 8 & 0,30 & \\
\hline & 9 & 0,25 & \\
\hline & 10 & 0,20 & \\
\hline & 11 & 0,15 & \\
\hline & 12 & 0,10 & \\
\hline & 13 & 0,08 & \\
\hline & 14 & 0,05 & \\
\hline & 15 & 0,04 & \\
\hline & 16 & 0,02 & \\
\hline
\end{tabular}

\subsection{Determinación del contenido de compuestos de cromo en el cemento portland}

Influencia del fundente

En la tabla 5 se incluyen los resultados obtenidos, correspondientes a diez determinaciones distintas, en la muestra de cemento $n .^{\circ} 6$ que se ha llevado a disolución por disgregación con $\mathrm{Na}_{2} \mathrm{CO}_{3}-\mathrm{K}_{2} \mathrm{CO}_{3}$ y con $\mathrm{LiBO}_{2}$. Los valores, media de diez lecturas concordantes, se han expresado en ppm y referido a la muestra seca a $105^{\circ}-110^{\circ} \mathrm{C}$.

T A B L A 5

Contenido de cromo, ppm

\begin{tabular}{|c|c|c|}
\hline Determinación & \multicolumn{2}{c|}{ Fundente } \\
\cline { 2 - 3 } & $\mathbf{N a}_{2} \mathbf{C O}_{3}-\mathbf{K}_{2} \mathbf{C O}_{3}$ & LiBO $_{2}$ \\
\hline 1 & 69,6 & 69,0 \\
2 & 75,0 & 71,0 \\
4 & 72,0 & 74,5 \\
5 & 45,0 & 61,5 \\
6 & 69,0 & 72,5 \\
7 & 70,0 & 69,5 \\
8 & 65,0 & 69,0 \\
9 & 64,4 & 67,0 \\
10 & 72,0 & 69,0 \\
\hline
\end{tabular}


Hecho el estudio estadístico y despreciando las medidas tomadas en la determinación 4, se obtienen para ambos casos los valores de la tabla 6, en donde puede apreciarse que con el fundente $\mathrm{LiBO}_{2}$ se tiene un valor medio $(70,1 \mathrm{ppm})$ ligeramente inferior que con el fundente $\mathrm{Na}_{2} \mathrm{CO}_{3}-\mathrm{K}_{2} \mathrm{CO}_{3}(70,6 \mathrm{ppm})$, pero con una desviación estándar estimada, $s$, una precisión, $\varepsilon$, y un coeficiente de variación $50 \%$ menores.

T A B L A 6

Características estadísticas

\begin{tabular}{|c|c|c|}
\hline \multirow{2}{*}{ Caracteristicas } & \multicolumn{2}{|c|}{ Fundente } \\
\hline & $\mathrm{Na}_{2} \mathrm{CO}_{3}-\mathrm{K}_{2} \mathrm{CO}_{3}$ & $\mathrm{LiBO}_{2}$ \\
\hline Media, $\bar{x}=\frac{\sum x}{N}($ ppm)) & 70,6 & 70,1 \\
\hline $\begin{array}{l}\text { Desviación estándar } \\
\text { estimada, s, }\end{array}=\sqrt{\frac{\sum(x-\bar{x})^{2}}{N-1}}$ & 4,42 & 2,23 \\
\hline Precisión, $\varepsilon=\frac{s \cdot m}{\sqrt{\sqrt{N-1}}}$ & 3,6 & 1,8 \\
\hline $\begin{array}{l}\text { Intervalo de confianza }(\mathrm{ppm}) \\
\text { Coeficiente de variación }=\frac{s \cdot 100}{\bar{x}}\end{array}$ & $70,6 \pm 3,6$ & $70,1 \pm 1,8$ \\
\hline
\end{tabular}

\subsection{Contenido de cromo, ppm, de 10 muestras de cemento portland}

En la tabla 7 se incluye el contenido de compuestos de cromo, expresados como cromo en ppm, referidos a la muestra seca a $105^{\circ}-110^{\circ} \mathrm{C}$. Cada muestra se ha analizado por duplicado y cada valor es la media de 10 lecturas concordantes.

T A B L A 7

Contenido de cromo total, ppm

\begin{tabular}{|c|c|c|c|}
\hline & \multicolumn{2}{|c|}{ Determinación } & \\
\cline { 2 - 3 } Muestra & $\mathbf{1}$ & Valor medio \\
\hline 1 & 90,6 & $\mathbf{2}$ & \\
\hline 2 & 50,0 & 92,5 & 91,5 \\
3 & 32,0 & 51,0 & 50,5 \\
4 & 55,2 & 32,5 & 32,3 \\
5 & 72,0 & 57,0 & 56,1 \\
6 & 69,6 & 70,5 & 71,3 \\
7 & 74,8 & 69,5 & 69,6 \\
8 & 47,0 & 76,0 & 75,4 \\
9 & 24,0 & 48,1 & 47,6 \\
10 & 53,6 & 24,0 & 24,0 \\
& & 54,5 & 54,0 \\
\hline
\end{tabular}




\subsection{Determinación de los compuestos de cromo (III) y cromo (VI) en un cemento portland}

Teniendo en cuenta las propiedades analíticas del $\mathrm{Cr}$ (III) y del $\mathrm{Cr}$ (VI), bien al estado de $\mathrm{CrO}_{4}{ }^{2-}$ o de $\mathrm{Cr}_{2} \mathrm{O}_{7}{ }^{2-}$, se puede conseguir la separación cuantitativa del $\mathrm{Cr}$ (III), precipitándolo como $\mathrm{Cr}(\mathrm{OH})_{3}$ a $\mathrm{pH}$ controlado, del $\mathrm{Cr}$ (VI).

La separación del $\mathrm{Cr}$ (III) se efectúa en el cemento, conjuntamente con los hidróxidos de los cationes Fe (III), Al (III), Ti (IV) y Sr (II). El precipitado, separado de la disolución que contiene entre otros iones los de $\mathrm{Cr}$ (VI), una vez purificado por lavado se lleva a disolución por ataque con ácidos minerales; de este modo tenemos separados el cromo (III) y el cromo (VI), en donde se puede proceder como se ha mencionado en los apartados anteriores.

El Cr (III), separado del Cr (VI), presente en la disolución se oxida con $\mathrm{K}_{2} \mathrm{~S}_{2} \mathrm{O}_{8}$ en presencia de Ag (I) y se elimina la interferencia del Fe (III) por precipitación del $\mathrm{Fe}(\mathrm{OH})_{3}$; a continuación, se forma el complejo con la s-difenilcarbacida y se determina la transmitancia (\%). Por otra parte, en la disolución que tiene el $\mathrm{Cr}$ (VI) se determina éste midiendo el valor de la transmitancia (\%) del complejo formado con el reactivo s-difenilcarbacida.

Otro procedimiento sería determinar en una parte alícuota de la disolución, que contiene la muestra del cemento el contenido total de cromo, como se ha mencionado en el apartado 4.5, y en otra parte alícuota proceder a la separación del $\mathrm{Cr}$ (III), por precipitación como hidróxido, del $\mathrm{Cr}$ (VI) y determinar éste último como se ha mencionado anteriormente.

En la tabla 8 figuran los contenidos de $\mathrm{Cr}$ (III) y de $\mathrm{Cr}$ (VI), expresados en ppm, referidos a la muestra seca a $105^{\circ}-110^{\circ} \mathrm{C}$, de cinco muestras de cemento portland.

TA B L A 8

Contenido de $\mathrm{Cr}$ (III) $y$ de $\mathrm{Cr}$ (VI), en ppm

\begin{tabular}{|c|c|c|c|}
\hline & \multicolumn{3}{|c|}{ Contenido de } \\
\cline { 2 - 4 } Muestra & Cr (III), ppm & Cr (VI), ppm & Cr (total), ppm \\
\hline 1 & 53,2 & 38,3 & 91,5 \\
4 & 20,0 & 36,1 & 56,1 \\
5 & 57,2 & 14,1 & 71,3 \\
9 & 24,0 & 0,0 & 24,0 \\
10 & 34,8 & 19,2 & 54,0 \\
\hline
\end{tabular}

\section{C O N C L U S I O N E S}

Primera: En el presente trabajo se prueba que el contenido total de compuestos de cromo (III y VI), así como de cromo (III) y de cromo (VI), que existen en el cemento portland, se puede determinar por espectrofotocolorimetría del complejo coloreado formado por reacción del cromo (VI) con la s-difenilcarbacida en medio $\mathrm{H}_{2} \mathrm{SO}_{4} \cdot 0,2 \mathrm{~N}$, con una desviación estándar estimada, $s$, de 2,23 y un coeficiente de variación de $3,18 \%$. 
Segunda: Asimismo, se demuestra que el empleo del $\mathrm{LiBO}_{2}$ como fundente da resultados análogos a cuando se emplea $\mathrm{Na}_{2} \mathrm{CO}_{3}-\mathrm{K}_{2} \mathrm{CO}_{3} ;$ sin embargo, la desviación estándar estimada, la precisión y el coeficiente de variación son menores, $50 \%$, así como la dispersión cuando se utiliza $\mathrm{LiBO}_{2}$.

Tercera: La concentración de cromo en la disolución de trabajo debe estar comprendida entre $0,1 \mathrm{mg} / l$ y $0,3 \mathrm{mg} / l$; para ello, o bien la cantidad de muestra debe responder a este requisito o tomar una parte alícuota apropiada de la mencionada disolución.

\section{B I B L I O G R A F I A}

(1) Valle, J.: Tesis Doctoral, A-60; Fac. Ciencias. Univ. Complut. Madrid (marzo, 1966).

(2) KEIL, F.: Cemento. Fabricación-Propiedades-Aplicaciones; Edit. Técnicos Asociados, S. A. Barcelona (1973), págs. 72-73.

(3) KleINBeRg, J.: Química Inorgánica; Edit. Reverté. Barcelona (1963)

(4) Cotton y Wilkinson: Química Inorgánica Avanzada. Edit. Limusa. Wiley. México (1967).

(5) Burriel, F.; Lucena, F. y Arribas, S.: Química Analítica Cualitativa. Edit. Paraninfo, Madrd (1957), págs. 217-221.

(6) Charlot, G.: L'analyse qualitative et les réactions en solution; Edit. Masson et Cie, París (1963), págs. 232-236.

(7) Hartford, W. H.: Treatise on Analytical Chem. Part. III, vol. 8, section A; Edit. Kolthoff, J. M.; Elving, Ph. J. and Sandell, E. B.; Intersc. Publ. New York, London (1963), págs. 237-337.

(8) Voinovitch, J. A.; Debras-Guedon, J. et Louvrier, J.: L'analyse des silicates; Edit. Hermann. París (1962), pág. 337.

(9) Bennet, H. and Hawley, W .G.: Methods of Silicate analysis. Edit. Acad. Press. Londres (1965), páginas $86-89$

(10) Kolthoff, I. M.; Sandell, E. B.; Meeham, E. J. y Bruckenstein, S.: Análisis Químico Cuantitativo. Edit. Nigar, S. R. L.; Buenos Aires (1972), pág. 879.

(11) Fischer, R. B. y Peters, D. G.: Análisis Químico Cuantitativo. Edit. Interamericana (1970), páginas 587-589 y 605-606.

(12) JAKOB, J.: Guía para el análisis Químico de las Rocas. C. S. I. C. Madrid (1944), págs. 102-105.

(13) Burriel, F.: Anal. Chim. Acta, 17, 545 (1957).

(14) Pinta, M.: Recherche et Dosage des eléments tracers. Edit. Dunod. París (1962), págs. 165-166.

(15) Duche, J.: Anal Chem. 33, 239 (1961).

(16) Charlot, G.: Colorimetric determination of elements. Edit. Elsevier. Amsterdam (1964), págs. 206-207.

(17) Standard Methods of Chem. Anal.: Vol. 1, sixth Edit. by Furman, D. Van Nostrand Co. Inc.; Princeton, New Yersey (1962), págs. 350-376.

(18) Flatt, R. and Cusani, P.: Anal. Chim. Acta, 21, 181 (1959).

(19) Bose, M.: Anal. Chim. Acta, 10, 201-109 (1954).

(20) Pflaum, R. T. and Howick, L. C.: J. Amer. Chem Soc., 78, 4862, (1956).

(21) Lichestein, I. E. and Allen, T. L.: J. Am. Chem. Soc., 81, 1.040, (1959).

(22) BaLt, S. and VAN DaLen, E.: Anal. Chim. Acta 25, 507, (1961); 27, 188, (1962), 29, 466, (1963); 30, 434, (1964); según Marczenko. loc. cit.

(23) MarcZenko, Z.: Spectrophotometric Determination of Elements; Chapter 17: Chromium; Edit. Ellis Horwood, Ltd. Chichester, Sussex, England (1976), págs. 213-223.

(24) Kolthoff, I. M.; Sandell, E. B.; Mecham, E. J. y Bruckenstein, S.: Loc. cit., págs. 1.086-1.091.

(25) K KaTZ, S. A.; MC NABB, W. M. and HAZEL, J. F.: Anal. Chim. Acta vol. 25, 2, 193-199, (1961).

(26) DEGRe, P.: Rev. des Mat. 685, 33-36, (1973).

(27) Feldman, F. and Purly, W. C.: Anal. Chim. Acta, 33, 273, (1965).

(28) Tenoutasse, N.: Rev. des Mat. de Const. 664-665, 3, (1971).

(29) Frfield, F. W. and $\mathrm{K}_{\mathrm{EAL}} \mathrm{y}, \mathrm{D}$.: Analytical Chemistry. Published by Intern. Textbook Co Ltd; London (1975), pág. 234

(30) Skoog, D. A. y West, D. M.: Análisis instrumental. Edit. Interamericana. 1. a Edición en español (1975), pág. 130.

(31) VogeL, A. I.: Quantitative Inorganic Analysis; Longman Group Limited. London (1972), págs. 791792.

(32) PCCH-64: IETCC. Madrid (1964), págs. 39-45.

(33) Tenoutasse, N.: loc. cit., pág. 65.

(34) Bononi, F.; Francardi, M. T. e Massazza, F.: Il Cemento, 3, 163-178 (1972).

(35) Crow, R. F. and Connoliy, J. D.: J. of Test. and Eval. ITEVA, vol. 1, 5, 382-393. (1973).

(36) VogeL, A. I.: loc. cit págs. 580-581.

(37) Vornovitch, I. A. y Colaboradores: Loc. cit. págs 146-152.

(38) Groves, A. W.: Silicate Analysis; West Norfolk Newps. Ltd. London (1951), págs. 48-51.

(39) Kolthoft, I. M. y Colaboradores: loc. cit., págs. 675-681. 